\title{
Sodium Glucose Co-Transporters 2 Inhibitors are Useful Addition for Treatment of Heart Failure with Reduced Ejection Fraction
}

\author{
Nasser Mikhail* \\ David-Geffen Medical School, USA \\ *Corresponding author: Nasser Mikhail, Endocrinology Division, Clinical Professor of Medicine, David-Geffen Medical School, CA, USA \\ To Cite This Article: Nasser Mikhail, Sodium Glucose Co-Transporters 2 Inhibitors are Useful Addition for Treatment of Heart Failure with Reduced \\ Ejection Fraction. 2020 - 7(3). AJBSR.MS.ID.001151. DOI: 10.34297/AJBSR.2020.07.001151.
}

Received: 阱 January 30, 2020; Published: 眥 February 18, 2020

\begin{abstract}
Background: Recent well-designed trials have shown that sodium-glucose co-transporter 2 (SGLT2) inhibitors decrease heart failure hospitalization (HFH) in patients with or without type 2 diabetes.

Methods: Review of literature (English, French, Spanish) from January 1990 to January 21, 2020. Key words included heart failure, sodiumglucose co-transporter 2, SGLT2 inhibitors, safety, randomized trials, and meta-analysis. Expert opinions and guidelines are also reviewed.

Results: The use of SGLT2 inhibitors in patients with type 2 diabetes was associated with significant relative reduction in HFH by 27-35\%. The latter reduction is most likely a class effect and is consistent in patients with various degrees of cardiovascular (CV) risk at baseline. In patients with heart failure and reduced ejection fraction (HFrEF), dapagliflozin decreased risk of a composite outcome of worsening heart failure (HF) or CV death by $26 \%$, as well as the secondary outcomes of HFH by $30 \%$ and death from any cause by $17 \%$. Moreover, dapagliflozin decreased severity of symptoms of heart failure. Importantly, the amelioration of previous outcomes was similar in patients with or without diabetes. Dapagliflozin did not cause major hypoglycemia in non-diabetic patients with heart failure. However, patients with advanced HFrEF with New York Heart Association (NYHA) class IV were not included.
\end{abstract}

Conclusion: SGLT2 inhibitors should be added to the standard care in most patients with HFrEF in presence or absence of type 2 diabetes.

Keywords: Heart failure; Sodium glucose cotransporter 2; Type 2 diabetes; Empagliflozin; Canagliflozin; Dapagliflozin

\section{Introduction}

SGLT2 inhibitors are medications approved to treat type 2 diabetes. They decrease hyperglycemia independently of insulin by lowering the renal threshold for glucose and therefore increasing urinary excretion of glucose [1]. Recent large CV trials have consistently shown that these agents decrease HFH by $27-35 \%$ compared with placebo [2-4]. HFrEF is defined as HF syndrome with left ventricular ejection fraction (LVEF) $\leq 40 \%$ [5]. A large randomized trial, called the DAPA-HF Trial, published in late 2019 demonstrated that dapagliflozin significantly decreased $\mathrm{HFH}$ and mortality in patients with HFrEF [6]. The results of previous trials were not reflected on American practice guidelines because they were published after the release of Practice guidelines from the American College of Cardiology/American Heart Association
(ACC/AHA) [7]. The Heart Failure Association of the European Society of Cardiology (ESC) Consensus published in August 2019 reports that there is enough evidence to consider that the ability of SGLT2 inhibitors to prevent HFH is a class effect. Yet, there is insufficient evidence to extend this observation to reductions in either CV or all-cause mortality or to patients without diabetes [8]. However, the latter Consensus was published after the release of the DAPA-HF Trial [6]. The author expects that the newer American and International guidelines will recommend SGLT2 inhibitors for standard management of most patients with HFrEF irrespective of existence of diabetes. However, the recently released guidelines of the American Diabetes Association (ADA) in 2020 mentioned that: "In patients with type 2 diabetes and established heart failure, a 
SGLT2 inhibitor may be considered to reduce risk of heart failure hospitalization" [9]. The ADA graded the latter recommendation as grade C, i.e. supportive evidence is derived from poorly controlled or uncontrolled studies [9]. In this mini review, the author presents data showing prevention of HFH in patients with type 2 diabetes in both randomized and real-world studies by SGLT2 inhibitors and effective treatment by these agents of actual HFrEF in patients with and without diabetes.

\section{Large Randomized Cardiovascular Trials of SGLT2 Inhibitors}

Upon request of the Federal Drug Administration (FDA), pharmaceutical companies are required to design large clinical trials to prove CV safety of their products. Thus, 3 large randomized double-blind trials were published to examine CV safety of the 3
SGLT2 inhibitors empagliflozin, canagliflozin, and dapagliflozin (table 1) [2-4]. The primary outcome in these trials was a composite of major adverse CV outcome (MACE) defined as CV death, non-fatal myocardial infarction or ischemic stroke. One of the secondary outcomes of these trials was HFH [2-4]. Despite the variable baseline $\mathrm{CV}$ risk across the 3 trials, the relative reduction in $\mathrm{HFH}$ compared with placebo was robust and consistent, ranging from $27 \%$ to $35 \%$ (table 1) [2-4]. A meta-analysis of the 3 studies estimated the overall risk reduction to be $31 \%$ (hazard ratio 0.69 , 95\% CI 0.61-79) [10]. The decrease in HFH was similar whether patients had established CV disease at baseline or had only CV risk factors [10]. Regarding the time course of events, the difference in rates of HFH between the SGLT2 inhibitors and placebo was evident early few months after starting treatment [2-4].

\begin{tabular}{|c|c|c|c|}
\hline \multicolumn{2}{|c|}{ Table 1: Hospitalization due to heart failure in major randomized cardiovascular trials of SGLT2 inhibitors in patients with type 2 diabetes. } \\
\hline Trial & EMPA-REG [2] & CANAVAS [3] & DECLARE [4] \\
\hline SGLT-2 inhibitor & Empagliflozin & Canagliflozin & Dapagliflozin \\
\hline Patients & $\begin{array}{c}\mathrm{n}=7020 \text {, mean age 63, 28\% } \\
\text { women } \\
\text { women }\end{array}$ & $\begin{array}{c}\text { N=10142, mean age 63, 35.8\% } \\
\text { N=17160, mean age 64, } \\
37.5 \% \text { women }\end{array}$ \\
\hline Patients with *CVD at baseline & $100 \%$ & $14.40 \%$ & $40 \%$ \\
\hline Patients with heart failure at baseline & $10.10 \%$ & Mean 188 wks, median 126 wk & Median 4.2 y \\
\hline Follow-up & $\begin{array}{c}\text { Median treatment 2.6 y, median } \\
\text { observation 3.1 y }\end{array}$ & $\begin{array}{c}0.67 \text { (0.52-0.87), P value not } \\
\text { reported }\end{array}$ & $\begin{array}{c}0.73 \text { (0.61-0.88), P value not } \\
\text { reported }\end{array}$ \\
\hline $\begin{array}{c}\text { Relative risk reduction in **HFH (Hazard } \\
\text { ratio, 95\% CI) }\end{array}$ & 0.65 (0.5-0.85), P=0.002
\end{tabular}

Note: ${ }^{*} \mathrm{CVD}=$ Cardiovascular Disease ${ }^{* *} \mathrm{HFH}=$ Heart Failure Hospitalization

\section{Meta-Analysis of Randomized Trials}

Yang et al [11] performed a network meta-analysis of 91 randomized trials to examine the incidence of HF among different classes of anti-diabetic agents. They found that SGLT2 inhibitors were superior in terms of risk of HF to insulin (odds ratio $0.75,95 \%$ CI 0.62-0.91), dipeptidyl peptidase inhibitors (odds ratio $0.68,95 \%$ CI 0.59-0.78), glucagon-like peptide-1 (GLP-1) receptor agonist (odds ratio $0.65,95 \% \mathrm{CI} 0.54-0.78$ ), and thiazolidinediones (odds ratio $0.46,95 \% 0.27-0.77$ ) [11]. In the subgroup of patients with baseline HF, SGLT2 inhibitors were also superior to metformin in reducing risk of subsequent $\mathrm{HF}$ (odds ratio 0.75 , 95\% CI 0.58-0.95) [11].

\section{Results of Real-World Studies}

The benefits of SGLT2 inhibitors on HFH and death were clearly shown in observational studies of real-world practice. The CVDREAL Study is a large study comparing 154,528 patients with type 2 diabetes initiating SGLT2 inhibitor with similar number of matched patients initiating other glucose lowering drugs across 6 countries [12]. Only $13 \%$ of patients had known CVD at baseline. Compared with other anti-diabetic agents, treatment with SGLT2 inhibitors was associated with 39\% relative risk reduction (hazard ratio $0.61,95 \%$ CI $0.51-0.73$ ) in $\mathrm{HFH}$, the primary outcome, and $51 \%$ reduction (hazard ratio $0.49,95 \% 0.41-0.57$ ) in all-cause death, the secondary outcome [12]. This large study adds important information to CV benefits of SGLT2 inhibitors. First, it supports the fact that reduction in HFH is a class effect shared by various SGLT2 inhibitors. Second, it suggests that reduction in HFH and all-cause death extends to relatively young patients with type 2 diabetes (mean age was $57 \mathrm{y}$ ) at lower CV risk at baseline [12].

In another study using 2 commercial and one Federal data sources, Patorno et al. [13] showed that initiation of empagliflozin in real-world practice was associated with $50 \%$ reduction in $\mathrm{HFH}$ as compared with initiation of sitagliptin over a mean follow-up of 5.3 months, hazard ration 0.50 (95\% CI 0.21-0.91) [12]. Subgroup analysis by presence of baseline CV disease, history of heart failure, and gender yielded consistent results [13].

\section{SGLT2 Inhibitors as Treatment for Heart Failure in Patients with And Without Diabetes}

Two studies were recently published to evaluate SGLT2 inhibitors as treatment for patients with HF irrespective of presence of diabetes. The first was a small short-term randomized trial using empagliflozin $10 \mathrm{mg} / \mathrm{d}$ in patients with HFrEF (mean LVEF 26\%) [14]. After 12 weeks, significantly greater proportions of patients 
treated with empagliflozin experienced improvements in $\mathrm{HF}$ symptoms, functional status and quality of life as compared with placebo [14]. Results were the same whether patients had type 2 diabetes (166 of $263,62 \%$ ) or no diabetes (97 of $263,38 \%$ ) [14].

The second study is a landmark randomized trial, the DAPA-HF trial, that examined the effect of dapagliflozin in 4,744 patients with HFrEF with LVEF of $40 \%$ or less (table 2) [6]. The primary outcome was a composite of worsening HF (defined as hospitalization or an urgent visit resulting in intravenous therapy for HF) or cardiovascular death [6]. Over a median follow-up of 18.2 months, the relative reduction in primary outcome in the dapagliflozin group versus the placebo group was $26 \%$ (hazard ratio $0.74,95 \%$ CI 0.65 $0.85, \mathrm{P}<0.001$ ) [6]. In addition, there was significant improvement in quality of life at 8 months. Importantly, results in patients without diabetes, who constitute $55 \%$ of the study population, were like those with diabetes [6]. Furthermore, all individual components of the primary outcome were in favor of dapagliflozin. (table 2). The DAPA-HF provides strong evidence that SGLT2 inhibitors do not only decrease incidence of HF but also may serve as actual therapy for HFrEF irrespective of presence of diabetes [6].

\begin{tabular}{|c|c|}
\hline Design & Randomized, Double-Blind, Multi-Center, 2 Groups \\
\hline Patients & $\mathrm{n}=4744$, mean age $66 \mathrm{y} / \mathrm{o}, 23 \%$ females, \\
\hline Patients' disease & Heart failure with ejection fraction $\leq 40 \%$, NYHA Class II (67\%), III (32\%), IV (1\%). \\
\hline Percentage of patients with/without type 2 diabetes & $45 / 55$ \\
\hline Primary outcome & $\begin{array}{c}\text { Composite of worsening heart failure (hospitalization or an urgent visit resulting in intra- } \\
\text { venous therapy for heart failure) or cardiovascular death }\end{array}$ \\
\hline Median follow-up & 18.2 months \\
\hline Intervention & Dapagliflozin $10 \mathrm{mg} / \mathrm{d}(\mathrm{n}=2373)$, placebo $(\mathrm{n}=2371)$ \\
\hline $\begin{array}{l}\text { Risk reduction in primary outcome with dapagliflozin vs } \\
\text { placebo }\end{array}$ & Dapagliflozin 16.3\%, placebo $21.3 \%$, *HR 0.74 (95\% CI, 0.65-0.85), P<0.001 \\
\hline Death from any cause & Dapagliflozin 11.6\%, placebo 13.9\%, HR 0.83 (95\% CI 0.71-0.97) \\
\hline Death due to cardiovascular causes & Dapagliflozin 9.6\%, placebo 11.5\%, HR 0.82 (95\% CI 0.69-0.98) \\
\hline Hospitalization due to heart failure & Dapagliflozin 9.7\%, placebo $13.4 \%$ HR 0.70 (95\% CI 0.59-0.83) \\
\hline Worsening renal function & Dapagliflozin 1.2\%, placebo 1.6\%, HR 0.71 (95\% 0.44-1.16) \\
\hline $\begin{array}{l}\text { Percentage of patients with } \geq 5 \text { increase in the } \mathrm{KCCQ}^{* *} \text { at } \\
\qquad 8 \text { months }\end{array}$ & Dapagliflozin 58.3\%, placebo 50.9\%, odds ratio, 1.15 (95\% CI 1.01-1.23) \\
\hline Discontinuation of study drug due to adverse effects & Dapagliflozin $4.6 \%$, Placebo $4.9 \%$ \\
\hline
\end{tabular}

Note: *HR: Hazard Ratio. ** KCCQ: Kansas City Cardiomyopathy Questionnaire. Score is from 0 to 100 , with higher score reflects fewer symptoms. An increase in scoring of $\geq 5$ is considered clinically important [6].

\section{SGLT2 Inhibitors for Treatment of Acute Decompensated Heart Failure}

In a pilot randomized, double-blind trial $(n=79)$, Damman et al [15] evaluated the cardiac effects and safety of administration of empagliflozin $10 \mathrm{mg} / \mathrm{d}$ for 30 days in patients with or without diabetes admitted to the hospital because of acute HF. No significant effects were observed between the empagliflozin group and placebo group in terms of dyspnea, diuretic response, or length of stay. Meanwhile, empagliflozin reduced a combined outcome of in-hospital worsening $\mathrm{HF}$, HFH, or death at 60 days compared with placebo, 4(10\%) vs. 13 (33\%); P =0.014 [15]. No safety issues were reported with empagliflozin with respect to renal function or blood pressure [15]. These data are overall reassuring, but clearly further studies are needed to study SGLT2 inhibitors in the setting of acute heart failure.

\section{Mechanisms of Cardiac Benefits of SGLT2 Inhibitors}

Mechanisms underlying the reduction in HFH of SGLT2 inhibitors are not fully understood and are currently an area of active research. These mechanisms include changes in arterial stiffness and cardiac oxygen demand, and reduction in the following parameters: serum uric acid, tissue sodium, albuminuria, ambulatory blood pressure, and weight [16]. Regarding the relative importance of these mechanisms, several observations need to be considered. First, the fact that reduction in HFH occurred early within the first few months after administration of SGLT2 inhibitors suggests that diuresis and natriuresis by these agents may be early mechanisms involved to decrease the after load. Second, it is unlikely that the mild reduction in hemoglobin A1c values (approximately $0.2-0.4 \%$ less than placebo) is a significant factor. This notion is supported by the fact that patients without diabetes had similar magnitude of cardiac benefits [6]. Third, recent data do not suggest a positive inotropic action by SGLT2 inhibitors. Thus, using cardiac magnetic resonance imaging, Verma et al [17] showed amelioration of left ventricular mass index after 6 months of empagliflozin therapy, but no significant effect on ejection fraction [17].

\section{Safety of SGLT2 Inhibitors in Heart Failure}

Overall, SGLT2 inhibitors were well-tolerated as reflected by rates of discontinuation due to adverse effects that are close to 
placebo [2-4,6]. Likewise, rates of hypoglycemia, acute renal failure are either similar or less than placebo [2-4]. It was particularly reassuring that no major hypoglycemic episodes were reported among patients without diabetes [6]. However, there are 2 adverse effects shared by members of the class. First, genital fungal infections are the commonest adverse effect of SGLT2 inhibitors, particularly in women. Second, diabetic ketoacidosis occurred 2-10 times more frequently with SGLT2 inhibitors than placebo, but the absolute number of events was small. Increased risk of lower limb amputation and fractures previously reported in one large CV trial with canagliflozin [3], were not seen with empagliflozin and dapagliflozin $[2,4]$.

\section{Rationale for Adding SGLT2 Inhibitors to Standard Therapy of Heart Failure Irrespective of Diabetes}

Based on the preceding, SGLT2 inhibitors should be included as part of management of most patients with HFrEF irrespective of presence of diabetes based on the following: first the reduction in HFH is robust and consistent across a wide range of patients' characteristics [2-4]. Second, the magnitude of decrease in $\mathrm{HFH}$ is substantial, was already manifest after few months, and was demonstrated on top of standard treatment of HF [6]. Third, the decrease in HFH by SGLT2 inhibitors was similar in patients with and without diabetes [6]. Fourth, in addition to reduction in $\mathrm{HFH}$ and amelioration of symptoms of HF, dapagliflozin significantly reduced CV death and all-cause death [6]. Fifth, SGLT2 inhibitors did not cause major hypoglycemia in patients without diabetes [6].

\section{Limitation for the Recommendation of Using SGLT2 Inhibitors in All Patients with HFrEF}

Despite the encouraging results of dapagliflozin in reducing CV events in patients with HFrEF, patients with NYHA class IV, i.e. patients with more advanced heart failure, were not adequately studied [6]. Thus, less than $1 \%$ of patients involved in the DAPAHF trial pertained to NYHA class IV, whereas approximately $67 \%$ and $32 \%$ of patients pertained to class II and III, respectively [6]. Therefore, use of SGLT2 inhibitors cannot be recommended in patients with HFrEF class IV until this group of patients is thoroughly evaluated in terms of efficacy and safety.

\section{Conclusions and Current Directions}

Reduction of HFH by $27-35 \%$ is a robust class effect of the 3 SGLT2 inhibitors [2-4]. The relative risk reduction in HFH by these agents emerges few months after starting treatment and is similar in patients with established $\mathrm{CV}$ disease and patients with only $\mathrm{CV}$ risk factors. In subjects with established HFrEF of NYHA class II and III, dapagliflozin significantly decreased HFH, CV death and allcause death [6]. Moreover, these benefits extend to patients without diabetes [6]. Based on the available data, the author recommends using SGLT2 inhibitors as a standard therapy for patients HFrEF class II and III, irrespective of presence of diabetes. Randomized trials ( $n=17$ trials) are underway to examine the effects of different
SGLT2 inhibitors on clinical and safety outcomes in patients with HF. These trials include patients with and without type 2 diabetes, and with HFrEF as well as HF with preserved ejection fraction (HFpEF) i.e. $\mathrm{LVEF} \geq 50 \%$ [8]. Randomized studies are needed to specifically evaluate efficacy and safety of SGLT2 inhibitors in patients with more advanced HF (i.e. NYHA class IV). The results of these ongoing trials will likely have great impact on the pending American and International guidelines of HF practice.

\section{Conflict of Interest}

The author has no conflicts of interest to declare.

\section{References}

1. Mikhail N (2014) Place of sodium-glucose co-transporter type 2 inhibitors for treatment of type 2 diabetes. World J Diabetes 5 (6): 854859.

2. Zinman B, Wanner C, Fichett D, Bluhmki E, Hantel S, et al. (2015) for the EMP-REG OUTCOME Investigators. Empagliflozin, cardiovascular outcomes, and mortality in type 2 diabetes. N Engl J Med 373(22): 21172128.

3. Neal B, Perkovic V, Mahaffey KW, ZeeuwD, Fulcher G, et al. (2017) for the CANVAS Program Collaborative Group. Canagliflozin and cardiovascular and renal events in type 2 diabetes. N Engl J Med 377: 644-657.

4. Wiviott SD, Raz I, Bonaca MP, Mosenzon O, Kato ET, et al. (2019) for the DECLARE-TIMI 58 Investigators. Dapagliflozin and cardiovascular outcomes in type 2 diabetes. N Engl J Med 380(4): 347-357.

5. Meer P, Gaggin HK, Dec William (2019) ACC/AHA versus ESC guidelines on heart failure. JACC Comparison. J Am Coll Cardiol 73(21): 2756-2568.

6. McMurray JJV, Solomon SD, Inzucchi SE, Kober L, Kosiborod MN, et al. (2019) for the DAPA-HF Trial Committee and investigators. Dapagliflozin in patients with heart failure and reduced ejection fraction. N Engl J Med 381(21): 1995-2008.

7. Yancy CW, Jessup M, Bozkurt B, Butler J, Casey DE, et al. (2017) ACC/ AHA/HFSA Focused Update of the 2013 ACCF/AHA Guideline for the Management of Heart Failure: A Report of the American College of Cardiology/American Heart Association Task Force on Clinical Practice Guidelines and the Heart Failure Society of America. J Card Fail 23(8):628-651.

8. Seferovic PM, Ponikowski P, Anker SD, Bauersachs J, Chioncel O, et al. (2019) Clinical practice update on heart failure: pharmacotherapy, procedures, devices and patient management. An expert consensus meeting report of the Heart Failure Association of the European Society of Cardiology. Eur J Heart Fail 21(10): 1169-1186.

9. American Diabetes Association. Cardiovascular disease and risk management: Standards of Medical Care in Diabetes. Diab Care 2020 43(1): S111-S134.

10. Zelniker TA, Wiviott SD, Raz I, Im K, Goodrich IL, et al. (2019) SGLT2 inhibitors for primary and secondary prevention of cardiovascular and renal outcomes in type 2 diabetes: a systematic review and metaanalysis of cardiovascular outcome trials. Lancet 393(10166): 31-39.

11. Yang D, He X, Liang H, Zhang S, Zhong X, et al. (2019) Comparative outcomes of heart failure among existent classes of anti-diabetic agents: a network meta-analysis of 171,253 participants from 91 randomized trials. Cardiovasc Diabetol 18(1): 47.

12. Kosiborod M, Cavender MA, Fu AZ, Wilding JP, Khunti K, et al. (2017) Lower risk of heart failure and death in patients initiated on sodiumglucose cotransporter-2 inihibitors versus other glucose-lowering drugs. The CVD-REAL Study (Comparative Effectiveness of Cardiovascular Outcomes in New Users of Sodium-Glucose Cotransporter-2 Inhibitors). Circulation 136(3): 249-259. 
13. Patorno E, Pawar A, Franklin JM, Najafzadeh M, Deruaz Luet A, et al. (2019) Empagliflozin and the risk of heart failure hospitalization in routine clinical care. Circulation 139(25): 2822-2830.

14. Nassif ME, Windsor SL, Tang F, Khariton Y, Husain M, et al. (2019) Dapagliflozin effects on biomarkers, symptoms, and function status in patients with heart failure with reduced ejection fraction. Circulation 140(18): 1463-1476.

15. Damman K, Beusekamp JC, Boorsma EM, Swart HP, Smide TDJ, et al Randomized, double-blind, placebo-controlled, multicentre pilot study on the effects of empagliflozin on clinical outcomes in patients with acute decompensated heart failure (EMPA-RESPONSE-AHF). Eur J Heart Fail 1-10.
16. Lytvyn Y, Bjornstad P, Udell JA, Lovshin JA, Cherney DZ (2017) Sodium glucose cotransporter-2 inhibition in heart failure. Potential mechanisms, clinical implications, and summary of clinical trials. Circulation 136(17): 1643-1658.

17. Verma S, Mazer CD, Yan AT, Mason T, Garg V, et al. (2019) for the EMPAHEART Cardio-Link-6 Investigators. Effect of empagliflozin on left ventricular mass in patients with type 2 diabetes and coronary artery disease: The EMPA-HEART CardioLink-6 randomized clinical trial. Circulation 140(21): 1693-1702. 